\title{
SUEÑOS DE ROBOTS: ¿PODRÍAN LAS MÁQUINAS DESARROLLAR CONSCIENCIA?
}

\section{ROBOTS DREAMS: COULD MACHINES DEVELOP CONSCIOUSNESS?}

\author{
Walter Walker Janzen ${ }^{1}$ \\ Fundación Educacional Intraeduc, Chile \\ Nelson Campos Villalobos ${ }^{2}$ \\ Fundación Educacional Intraeduc, Chile
}

\begin{abstract}
Resumen: El presente artículo traduce el debate entre un matemático y un filósofo, que discuten -cada uno con una posición específica- sobre la imposibilidad o plausibilidad de la existencia de una inteligencia artificial de tipo humana en las máquinas y, sobre la emergencia de una consciencia en ellas. Un debate que surge dentro de una Universidad, al interior de su Facultad de filosofía, debido a la necesidad de abordar formalmente la cuestión. Posicionados en diferentes perspectivas, los autores concuerdan que los conceptos de mente, consciencia e inteligencia corresponden a la misma tendencia evolutiva y que la selección natural produjo en un continuum los procesos mentales que en nuestra especie emergen como más desarrollados o elevados y cuyo objeto ha sido la sobrevivencia.
\end{abstract}

Palabras clave: Evolución darwiniana, Robots, Consciencia, Inteligencia artificial.

\begin{abstract}
The present article, translates the debate between a mathematician and a philosopher who argue - each one with a specific positioning - on the impossibility or the plausibility of the existence of an artificial intelligence of the human type in the machines and, on the Emergence of a consciousness in them. A debate that arises within a university, within its philosophy faculty, due to the need to formally address the issue. Positioned the authors from different perspectives, both coincide in that the concepts mind, consciousness and intelligence, correspond to the same evolutionary trend and that natural selection has produced in a continuum the mental processes that in our species They arise as more developed or elevated and whose object has been survival.
\end{abstract}

Key words: Darwinian evolution, Robots, Consciousness, Artificial intelligence.

1 Doutor em Pedagogia e Mestre em Educação; Professor do Estado de Matemática e Estatística. Email:walterfwalker@gmail.com. https://orcid.org/0000-0002-9120-621X

${ }^{2}$ Doutor em Filosofia e Ciências da Educação pela Universidad Ramón Llull, Barcelona; Professor da Educação especial e diferenciada pela Pontificia Universidad Católica de Chile. E-mail: trackdos2@yahoo.cl. 


\section{Introducción}

¿Por qué presentar bajo la forma de un diálogo este trabajo? Porque así es como se gesta una investigación compartida, como la conversación ordenada para plantear con rigurosidad un problema de esta naturaleza. Este ir y venir de ideas es lo que llevó a los griegos a desarrollar la filosofía y de ahí la ciencia en la forma que ha perdurado por más de 25 siglos.

El presente debate surgió primeramente de la necesidad de contar con una base reflexiva que permita discriminar entre lo que las máquinas hacen en la actualidad, lo que es la robótica y lo que sería una verdadera inteligencia artificial (I-A), siguiendo una secuencia que se inició algunos años atrás ${ }^{3}$. Esto, para un eventual programa académico de la Facultad de Filosofía de la Universidad. En segundo término, por la petición de los estudiantes para que este debates e realizara públicamente en la comunidad universitaria, porque el tema es contingente, lo amerita y es bastante desconocido entre los estudiantes: las implicaciones del uso de inteligencia artificial y la eclosión de una consciencia no humana, no es una mera cuestión de hardware y software.

Como señalara Darwin, y en el tenor de este conversatorio, toda observación debe hacerse a favor o en contra de una hipótesis, si es que ha de servir para alguna cosa. Esta afirmación un poco renuente de Darwin por el método - que no es nuestro caso-, se explica operacionalmente porque

Las preguntas e hipótesis sobre las causas o función del comportamiento (animal) son el nivel menos empleado por Darwin, apenas alcanzan un 2\%. Este porcentaje no es del todo sorprendente debido a que Darwin no era un experto en la observación de la conducta y para aquella época la función de la conducta no se estudiaba sistemáticamente (PÉREZ et al. 2007, p.514)

En el fondo es lo que los autores de este artículo han hecho: reunirse para discutir sus propias hipótesis referidas a la I-A. Si bien los autores están en desacuerdo en varios puntos, ambos coinciden en que la teoría de Darwin ${ }^{4}$ explica muy bien los fenómenos que se estudian aquí. Está dirigido a un estudio epistemológico y a una reflexión filosófica sobre el tema de la I-A y la posibilidad o imposibilidad de las máquinas de llegar a tener una mente como la humana. Justificamos el empleo del término consciencia, porque si hablamos de conciencia podríamos entender que tiene componentes morales.

\footnotetext{
${ }^{3}$ Este es el tercer debate. El primero se refirió al estatus epistemológico de la Matemática y el segundo, al Teorema de Gödel y sus implicancias para el desarrollo de las Ciencias Sociales.

${ }^{4} \mathrm{El}$ origen de las especies (1859); El origen del hombre (1871)
} 
Cuando hablamos de máquinas inteligentes o de la Inteligencia Artificial, tenemos que recordar que los conceptos básicos de ese campo de estudio- mente, consciencia, inteligencia- constituyen parte de lo que llamamos conceptos indecidibles, porque no hay una sola teoría, sino muchas para tratar de explicar su contenido.

Estos tres constructos que son tan importantes para la filosofía y la psicología se han tratado generalmente en forma separada, por fines didácticos o de investigación, tanto desde la filosofía como desde la biología, lo cual ha originado una cadena de errores y confusiones en la amplia producción de textos y libros sobre estas materias. Por lo mismo, muchos conceptos, teorías e hipótesis se han tornado elusivos y nos han dejado como herencia múltiples equívocos. Por ejemplo, es necesario separar entre consciencia y autoconsciencia pues ambos términos son distintos y aplicarían en contextos evolutivos distintos.

\section{El Debate}

CV: Buenos días estimado doctor, vamos a iniciar esta discusión académica haciendo notar que la Inteligencia Artificial es un tema de alto interés para nuestra especie, por las implicaciones que ésta tiene o tendrá para todas las ciencias, ya sea para las "duras" como la física y la química, como para las ciencias sociales o las aplicadas, esto es, las ingenierías y las tecnologías de la computación...

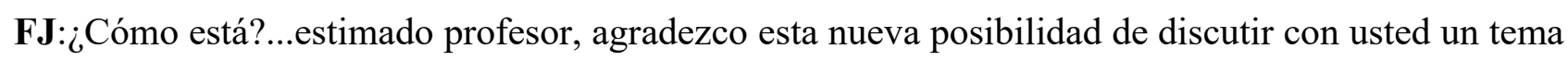
que convoca a una buena parte de nuestra comunidad universitaria...a estas alturas ambos sabemos que la realidad ha superado con creces las proposiciones iniciales de la ciencia-ficción...por eso estamos aquí, en este anfiteatro, con nuestros estudiantes...

CV: Bueno, a los dos se nos ha hecho evidente que se hace necesario esclarecer esta temática y reflexionar sobre una pregunta que antes que los filósofos, se hicieron los autores de ciencia ficción: ¿Podrán las máquinas adquirir mente, conciencia e inteligencia en forma autónoma? La respuesta a esta incógnita tendrá repercusiones sobre el futuro de la humanidad, por lo cual no es un tema menor al que atender. Si la respuesta es positiva se abre un camino de enorme progreso para el ser humano. Si es negativa habrá que seguir trabajando con máquinas complejas, pero no pensantes.

En este debate, partiremos por establecer hipótesis de trabajo: la primera, defendida por mí, es que la Inteligencia Artificial no podrá dar origen a una entidad capaz de razonar en forma autónoma, ni lograr consciencia ni de tomar decisiones tal como lo hace el cerebro humano.

Por su parte, doctor, tal como me lo manifestó previamente, usted se adhiere a la hipótesis contraria, 
es decir que las máquinas, con el tiempo, podrán desarrollar similares y superiores capacidades que las que posee el cerebro humano.

FJ: Así es...

CV: Como me ha correspondido abrir, trataré de, sin alargarme demasiado, presentar con claridad la problemática que trataremos...

Una preocupación que empezó hacia la séptima década del siglo pasado se refirió al temor plasmado en novelas y películas, que las máquinas robóticas pudiesen independizarse de sus creadores y amenazar a la supervivencia de la especie humana en el planeta. Pero no hubo planteamientos serios acerca de cómo las máquinas podrían desarrollar consciencia, toda vez que aún no se contaba con la suficiente base teórica ni con la experiencia necesaria. Hasta elpresentenosabemosconseguridadquéeslamente,niquéeslaconsciencia ni hemos definido con certeza a la inteligencia ni sabemos cómo se ha desarrollado lo que llamamos pensamiento. Pero desde la filosofía no tenemos impedimento alguno para reflexionar sobre este problema de un futuro relativamente cercano.

Creo que la psicología y las neurociencias seguirán entrampadas en su avance actual si no logramos dilucidar esas tres interrogantes y las disciplinas que se nutren de esos saberes; como la pedagogía, la sociología y la antropología, que tampoco podrán desarrollar en buena forma sus programas de investigación ni cumplir con sus objetivos y metas derivadas de sus estatutos epistemológicos.

Durante siglos hemos considerado que el hombre era el único poseedor de inteligencia, consciencia y de razonamiento en el mundo y por ello hemos tratado a las especies distintas a nosotros como inferiores. En el estudio de la zoología y de la evolución, no hay ningún antecedente que demuestre que hay base alguna para creer en alguna supuesta superioridad. Si entendemos a la inteligencia como la capacidad de supervivencia y si ésta es indicadora de éxito evolutivo, nuestra especie con apenas unos pocos millones de años de existencia no puede compararse con la capacidad de superar los obstáculos vitales que han presentado otras especies, como los selacimorfos (tiburones) que existen desde hace al menos 400 millones de años y que pueblan los mares desde el período Devónico. Los insectos dominan el planeta y su biomasa es superior a la de los humanos. No hay ninguna inferioridad en esos seres, sino al contrario, han demostrado ser capaces de propagar sus genes durante a lo menos 600 millones de años, desde la explosión del Cámbrico. Cuesta pensar que nuestra propia especie pudiera tener un logro similar.

Recién en el siglo XX las ciencias médicas aprendieron que compartimos nuestro genoma con el resto de los animales: 
"[...] podemos afirmar que todos los seres vivos comparten las mismas funciones biológicas básicas. De todos estos estudios ha surgido una visión unificadora de los procesos biológicos basada, en última instancia, en el proceso evolutivo. Los organismos tienen un origen común, como propusieron Darwin y Wallace, comparten el mecanismo de almacenamiento y liberación de la información genética basado en la universalidad de la función del ADN, del ARN y en el mecanismo de código genético. Por último, todos los componentes del Reino Animal comparten el mismo proceso genético de diseño corporal" (MORATA, 2014, p. 8).

Según Campos (2005), para cualquier estudioso de la evolución resulta indudable que hay una continuidad genética, pues como un ejemplo podemos decir que el Pax 6 es un grupo de genes que regulan la formación del ojo y está presente en los animales que poseen ojos, es decir, de prácticamente todos.

Lo mismo ocurre con los elementos bioquímicos de la visión. Somos parientes de cada una de las otras especies, porque tenemos un antecesor común; es significativo el que no hayamos querido verlo así. Como señalé al comienzo, no quisiera alargar mucho esta introducción, por lo que quisiera que el profesor nos de sus opiniones sobre el tema...

FJ: Antes que nada, profesor, tenemos que precisar qué entendemos actualmente por Inteligencia Artificial. Los antiguos chinos tenían un sabio proverbio que dice que la sabiduría comienza con el correcto uso de las palabras, por eso precisemos: en primer lugar, la I-A se refiere a la relación entre la mente humana y las máquinas para el estudio de los límites del comportamiento inteligente de las últimas $\mathrm{y}$, en segundo término, al estudio y práctica de sistemas computacionales que permitan realizar actividades automáticas que sean propias del cerebro humano.

Si queremos llevar la precisión conceptual a un nivel más elevado, podemos considerar que existen dos líneas teóricas. Madruga (2007) lo plantea claramente: la I-A “fuerte", que sostiene que la mente es una función del cerebro y que éste en sí es un simple ordenador (computador) y la I-A "débil", que simplemente espera que el avance de los ordenadores ayude a explicar de una vez por todas el funcionamiento del cerebro y entregue modelos para entender la mente y la consciencia.

Las esperanzas que se pusieron en la I-A no han tenido aún la compensación práctica esperada para la enorme cantidad de publicaciones a que dio origen, seguramente porque aún carecemos de suficiente información científica y tecnológica sobre el cerebro humano, que es el modelo para la investigación sobre la inteligencia aplicada a los ordenadores; lo mismo que ha ocurrido con la psicología cognitiva, que empezó con tantos bríos y actualmente está siendo reemplazada por la neurociencia, especialmente en el campo de la investigación educativa. Aun cuando hay autores que aseguran que hay avances significativos en las aplicaciones de la I-A, nada hay que acredite la existencia en el planeta de un solo robot que sea autónomo y que posea inteligenciaenlaformaenqueladescribimosevolutivamente.Porotrolado, no hay nada en las leyes científicas que conocemos y rigen nuestro universo, que digan que la inteligencia es solamente 
patrimonio de los seres nacidos vivos biológicamente...

CV: Veamos; quién empleó el término I-A por primera vez, fue el matemático John McCarthy quien en el año 1956 lo usó en una conferencia que dictó en New Hampshire. En esos años todo el mundo científico que trabajaba en computación conocía el trabajo de Alan Turing (1936), quien ha sido considerado como el inspirador de lo que McCarthy bautizó como Inteligencia Artificial y que llevó al surgimiento de otra disciplina: la robótica. Si bien las nuevas disciplinas que mencionamos datan de poco más de sesenta años atrás, hay antecedentes de que los griegos y algunos autores judíos de hace milenios ya tuvieron la preocupación de que podrían surgir creaciones del hombre parecidas a los robots. Por ejemplo, el mito del Golem describiría muy bien lo que a los ojos de los antiguos sería un robot. John Locke (1632-1704) consideraba que la mente tendría que venir antes que la materia, pues para él era impensable que la materia inconsciente pudiese producir un ser pensante e inteligente. Esa idea viene siempre a la mente de toda persona que reflexione sobre el tema, porque no es evidente cómo la materia inanimada puede producir algo tan distinto, tan delicado como es la vida y por supuesto la consciencia. Descartes creyó solucionar esa problemática separando la materia biológica (el cuerpo) de la mente, pero lo que hizo fue justamente producir una brecha enorme entre ambos conceptos.

La mente, esa propiedad emergente del sistema nervioso, estaba destinada a aparecer, aun cuando la espera fue en realidad muy larga. Si contamos desde el Cámbrico, cuando aparecen los animales multicelulares, la conciencia demoró prácticamente 600 millones de años en surgir. De todas maneras, era cuestión de tiempo y de sistemas nerviosos cada vez más complejos. Eso es la evolución, que nunca se ha detenido en el planeta. Por ejemplo, lo que ha ocurrido con el hombre, que no pudiendo continuar aumentando el tamaño de su cerebro ${ }^{5}$-por consideraciones anatómicas del paso de la cabeza por el canal del parto-, ello no ha afectado su supervivencia ni ha detenido el proceso de su evolución biológica y mental. Es más, ha aumentado sus niveles de inteligencia, esto es, sus capacidades de adaptación.

A mí me preocupa el cómo las máquinas, que están desconectadas de la mente humana, porque solamente reciben instrucciones del hombre, podrían predecir acontecimientos que son propios de nuestra mente. La otra pregunta que surge de lo anterior es ¿cómo podríamos saber cuándo una máquina tiene o adquirió consciencia? El test de Turing (1950) resulta insuficiente e ingenuo para dilucidar este aspecto. Rodrigo González (2007) ha escrito sobre los mitos y dogmas asociados...

FJ: ¿Podría precisar su opinión sobre el Test de Turing?

CV: Alan Turing pensaba que el test consistía en una conversación entre una persona y una

${ }^{5} \mathrm{Al}$ parecer no existe una correlación biunívoca entre volumen y desarrollo: el hombre de cro-magnon tendría alrededor de 200 centímetros cúbicos más de masa cerebral que el homo sapiens.

Revista Tópicos Educacionais, Pernambuco, v. 27, n. 02, p. 164-181, 2021. ISSN: 2448-0215.

https://periodicos.ufpe.br/revistas/topicoseducacionais/index

DOI: $10.51359 / 2448-0215.2021 .249287$ 
computadora y si la persona no se daba cuenta que estaba hablando con una máquina entonces significaría que se estaría en presencia de una inteligencia por parte de la máquina o que la máquina podía pensar. Es evidente que esa no sería la demostración de que la máquina posee consciencia o que puede pensar. Solamente podríamos decir que el programa del computador es bueno para simular respuestas aparentemente legítimas en una conversación no dirigida por el autor del programa. Por otra parte, Turing pensaba que el cerebro humano se asemejaba a un computador que fuese de propósito general, que tuviese un programa incorporado. Esa máquina ideal también es llamada computador abstracto, por la idea que subyace tras este tipo de instrumento cibernético. Los insectos, por ejemplo, serían máquinas de propósito especial y la evolución no les daría nunca un tipo de mente pensante, así como no esperamos -por ahora- que un PC desarrolle consciencia.

FJ: Bueno, Turing hizo mucho más de lo que usted describe, pero creo que como nos estamos introduciendo ya en el tema que nos preocupa es prudente que podamos hacer algunas definiciones conceptuales. Por ejemplo, necesitamos precisar qué entendemos por mente, por consciencia y por inteligencia antes de irnos a la discusión propiamente de si las máquinas podrán algún día tener propiedades como las del cerebro humano y lograr una autonomía en cuanto a dirigir su propia evolución. Quizás usted profesor podría hacernos un resumen de cómo han surgido esas propiedades en nuestra especie. Como estamos empezando a introducirnos en un tema que es filosófico, me agradaría doctor que nos informara sobre las raíces del problema.

CV: Como residuo de la gran división cartesiana, para la mayor parte de los autores la mente es una propiedad del cerebro que se da en exclusividad en nuestra especie, lo cual no es cierto, porque si de verdad es un producto cerebral, entonces, los otros animales que también poseen cerebros tienen que tener una mente que contenga todas las habilidades de las cuales es capaz el animal. El problema está en la calidad de los cerebros y en la calidad de las mentes que correspondan. A mayor calidad cerebral, mayor es la calidad de la mente, entendiéndose esta como la capacidad de producir pensamiento y consciencia de esos fenómenos, debido en parte a la evolución genética.

En resumen, pienso, luego soy consciente de mi mente y, por tanto, en este momento crucial de la historia humana, emerge la reflexibilidad y aparece el Yo.

Como sé que usted ha estudiado a Descartes, me gustaría que compartiera lo que ha llegado a conocer sobre el problema mente-cuerpo, que creo que aún no tiene solución, ni aún con el esperado aporte de las neurociencias, a diferencia de lo que sugiere José Ramón Alonso en el 2014...

FJ: De acuerdo, veamos la argumentación de Descartes con alguna precisión: la esencia de la materia es su espacialidad, es decir, su extensión espacial. Entonces, como la mente por intuición y por definición es una sustancia que carece de espacialidad, es distinta a la materia o, mejor dicho, no tiene las cualidades categoriales propias de la materia. Hecha la distinción, la mente reside en el 
cerebro, pero no es el cerebro; actúan ambos en una relación causal y la mente produce estados mentales que hacen, por ejemplo, que el cuerpo se mueva según la voluntad lo desee. Esa relación cuerpo-mente es de doble vía, de lo mental a lo físico y viceversa.

Desde un punto de vista histórico, podemos definir al dualismo cartesiano como la doctrina de la interacción psico-física, bidireccional. Es altamente probable que la espacialidad para Descartes era la definida por $\mathrm{R}^{3}$, esto es, el espacio tridimensional real, pues en su siglo todavía no se desarrollaba el álgebra asociada a la geometría n-dimensional. Descartes pronto se dio cuenta que en su línea de pensamiento tendría que encontrar un intermediario entre el alma o mente y el cuerpo o estaría en un problema sin solución o al menos circular, lo que prontamente corrigió señalando que el alma (la mente) tenía un lugar en el cerebro, en la glándula pineal, con lo cual el problema quedaba superado. La biología no le dio la razón a Descartes. Algunos autores consideran, como Damasio (2001), que el gran filósofo habría incurrido en un error lógico, como había señalado tempranamente.

Ryle, en 1949, quiso equiparar dos categorías distintas -el cuerpo y el alma- lo cual aparentemente no es lógicamente posible. Por mi parte, no creo que Descartes cometiera ese error. Él era un gran lógico y conocía perfectamente las categorías aristotélicas. Descartes trató de conciliar más bien sus convicciones científicas con la posición teológica en boga en su tiempo.

Desde una perspectiva filosófica, quizás lo que aplica en Descartes es la búsqueda de un principio escolástico, el fundamentum divisionis (fundamentos para establecer una distinción) que consiste en ubicar en categorías distintas a dos elementos separados en la realidad -que existen realmente- pero que pueden también categorizar a cosas que no pueden existir separadamente por lo que hay necesariamente un punto de unión entre ellas. Cuestión que la matemática moderna resolvió posteriormente con mucha precisión.

Entonces, Descartes separa a la mente del cuerpo, pero entiende que hay una unión en esas categorías, si bien pueden funcionar separadamente, como consideran los creyentes, que cuando el cuerpo muere, el alma sigue su propia trayectoria ontológica.

Habiendo llegado a este punto, y al parecer sin mayor controversia, quisiera que usted, profesor, expusiera sus ideas sobre la emergencia de la mente, de la conciencia y sobre la inteligencia en nuestra especie. Porque sin esa base teórica resulta difícil adherir a una hipótesis para apoyar o negar la idea de la posible emergencia de la consciencia en los robots...

CV: Trataré de explicar brevemente mis ideas al respecto de los conceptos de mente, consciencia y pensamiento.

La mente pareciera ser una entidad emergente, en el sentido filosófico de poseer propiedades que son distintas y también irreducibles a los procesos de sus partes constituyentes. En este sentido, 
la mente es distinta al cerebro que origina su existencia. Tenemos que recurrir a la antigua idea que el todo es mayor que la suma de sus componentes. Dicho lo cual tenemos que trasladarnos a un plano distinto, fenomenológicamente hablando. Esto porque la emergencia de nuevas funciones cerebrales en la evolución tiene que llevar a nuevas propiedades que no son distintas, porque la emergencia es simplemente una propiedad-de-las-propiedades, como diría Heidegger (GARRIDO, 2015). El cerebro transduce la información biológica en un ente subjetivo contenido en la mente y entendible por ésta. La mente es el reservorio, el empaque de las cualidades psíquicas que conforman a un ser viviente y que le dan el sello de ser diferente a los demás de su especie si bien es parte de ella con propiedades comunes a los demás miembros. La mente y la consciencia no emergieron repentinamente en el cerebro humano, sino que sus antecedentes están en las otras especies no humanas. Existe evidentemente un continuum entre las especies más simples a las más complejas. Es lo que llamamos evolución darwiniana.

Para algunos autores, mente equivale a entendimiento, es decir, a la facultad de pensar.

Según lo dicho, para entender a las otras mentes, incluidas las de los animales, tenemos que reconocer que estamos ante un problema de la calidad de las mentes, que distintas a nosotros, pueden ser semejantes, pero de otra calidad. Pero todas son resultantes de la evolución.

FJ: Por mi parte, si me permite esta interrupción, hay que agregar que calidad es un término filosófico, pues contiene una relatividad conceptual que complica el entender el tema, aunque hoy la cibernética, la teoría de probabilidades y la lógica difusa ${ }^{6}$ se ocupen estrechamente de la calidad. Por eso, definamos por ahora, calidad como el grado mejor o superior que alcanzan los organismos a medida que evolucionan. Si aceptamos esta definición nos encontramos con una pregunta nueva: ¿Qué sucederá si logramos crear máquinas cada una con mil o diez mil veces la capacidad de un cerebro humano y además con consciencia?, ¿querrían estar sometidas a nuestro dominio?

CV: Estoy muy de acuerdo con su alcance, es justamente lo que en otras palabras trataba de decir. Nos puede suceder como en el cuento del aprendiz de brujo, que no puede controlar los sucesos que su magia incipiente e inexperta está produciendo. La evolución demoró, como dijimos antes, desde el periodo Cámbrico cuando aparecen los animales multicelulares, más de 600 millones de años en generar mentes totalmente conscientes. Es un camino muy largo el recorrido y en cambio las máquinas son recientes. Quizás el camino que nos llevó a la mente humana ha sido una simple casualidad entre miles y miles de posibilidades, salvo que pensemos que existe un creador o una supermente capaz de efectuar un diseño tan complejo. Pero aun así se recorrió un largo camino para llegar a la mente consciente. ¡Nada menos que 4.500 millones de años!

\footnotetext{
${ }^{6}$ Véase el trabajo matemático de Zadeh (1973)
} 
En términos corrientes, la mente es el contenedor, es el reservorio de los conocimientos y habilidades intelectivas que produce el cerebro, en calidad y cantidad. Como decía Descartes, el Yo Pienso es la mente. Este contenedor es consciente de su propia existencia. El cognoscente se conoce a sí mismo en una reflexibilidad continua. La mente es entonces un epifenómeno, un fenómeno secundario o derivado de otro fenómeno principal o determinante, cuyo origen indudable está en el sistema nervioso, pero que recién estamos entendiendo cómo se produce esa transformación.

FJ: Usted tiene la razón profesor; la materia inanimada o muerta no nos puede decir nada sobre la mente ni sobre la consciencia. Hay que buscar en otras formas de investigación y por eso las neurociencias van por el buen camino para descifrar, en el sistema nervioso vivo, la emergencia o como la llama usted, la transducción de la mente desde un conjunto enorme de neuronas a una mente que tiene la propiedad del Yo, que recuerda y conoce la realidad y se hace preguntas sobre el universo que aún no estamos en condiciones de responder. Pero también hay que tomar en cuenta que la ciencia avanza y quizás las esperanzas puestas en las computadoras fotónicas o cuánticas, ambas con una enorme capacidad de memoria y una infinita velocidad de procesamientos, podrían hacer real el sueño de los robots.

CV: Como el tiempo siempre pasa velozmente en nuestra actividad académica, trataré de resumir lo esencial sobre la mente: es una construcción de nuestro genoma, que transduce lo físico en pensamiento, que es por supuesto subjetivo, ajeno a la física; es una emergencia, es decir, algo más que la materia que conforma el cerebro; es un reservorio de los hechos estrictamente psicológicos que se producen en el cerebro; no todas son conscientes ni nuestra mente es consciente en todo momento, como lo planteó Sigmund Freud ${ }^{7}$; su nacimiento y desarrollo se produce mediante la evolución darwiniana de las especies; el fenómeno evolutivo que la originó se debe replicar muchas veces en el universo porque ninguna ley de la física o de la biología lo impide, no así la consciencia o la inteligencia que reflexiona sobre el universo, que debe ser más escasa por la calidad de cerebro que se requiere para ese logro.

Por último, el estudio del cerebro y de sus componentes no nos permite conocer ni explicar los procesos subjetivos del sistema nervioso, así como tampoco el estudio de esos tejidos nos puede explicar la consciencia.

FJ: ¿Todo esto porque usted quiere decir que los robots tendrán que desarrollar una mente para tener autoconsciencia, librarse del determinismo a que los someten los programas creados por el ser humano y para que puedan funcionar?...

CV: Esa mente robótica se produciría si los autómatas pudiesen liberarse de la programación a que las somete el hombre. Una máquina dotada de I-A autónoma y plena podría considerarse una

\footnotetext{
${ }^{7}$ En su teoría de la personalidad

Revista Tópicos Educacionais, Pernambuco, v. 27, n. 02, p. 164-181, 2021. ISSN: 2448-0215.

https://periodicos.ufpe.br/revistas/topicoseducacionais/index

DOI: $10.51359 / 2448-0215.2021 .249287$
} 
máquina inteligente. Esta parte de la conversación me recuerda lo que dijo Geymonat sobre que se podía deducir del teorema de Gödel una aplicación filosófica importante; “[...] la afirmación de la imposibilidad de sustituir completamente el trabajo del cerebro humano por el de computadoras, al menos tal como es posible concebirlas hoy en día" (2009, p. 642).

Por mi parte, creo que surgirá una nueva tecnología que nos libere de la metáfora que asimila la I-A al sistema nervioso humano. Quizás hemos equivocado el camino y si bien el funcionamiento del cerebro humano puede explicar en parte al computador, éste no puede explicar en nada al cerebro humano. Sigo pensando que una aproximación a la I-A plena podrá concebirse, pero bajo nuevos fundamentos teóricos que aún no existen.

FJ: Si creo haber entendido bien, no solamente necesitaríamos que los robots cuenten con una mente, sino lograr que esa mente les permita adquirir consciencia. Con esto podría decirse que jamás entonces los robots podrán adquirir una autonomía. Pero no todos los científicos están de acuerdo con esa posición intelectual. Creo que no debemos ser pesimistas, porque como usted sabe, en la historia de la ciencia conocemos muchos casos en que reconocidos científicos dijeron que el hombre jamás podría volar o que la velocidad de los vehículos de motor mataría a sus conductores. El mismo Einstein dijo haberse equivocado al postular una constante que impediría la expansión del universo. Creo doctor que sería interesante que diese los argumentos que según usted valida a la hipótesis de la imposibilidad de la plena I-A.

CV: Primeramente, tendría que decir que una plena consciencia significa disponer de libre albedrío. Y éste está limitado por la moral, en el caso del ser humano. Si las máquinas son distintas a nosotros, entonces no veo cómo podrían lograr el libre albedrío con una moral humana. Quizás las máquinas tendrían una moral muy distinta a la nuestra porque estaría centrada en ellas y no en el hombre. En segundo lugar, tenemos que tomar muy en cuenta los descubrimientos de Gödel. En un debate anterior (WALKER y CAMPOS, 2017, pp. 13-14), en esta misma universidad, se señaló lo siguiente y que es adecuado citar aquí porque nos aclara mucho las ideas que estamos tratando:

Si las proposiciones que son fundamentales de todo sistema, sea este científico, social, psicológico, biológico o matemático, fuesen de tipo axiomático, significaría un golpe mortal para la llamada I-A (inteligencia artificial), toda vez que las máquinas solamente pueden deducir un enunciado de otros ya existentes; en virtud del teorema las máquinas no pueden comprender aquello ajeno a los axiomas que conocen. Esto porque están sometidas a un determinismo tal que sus procesadores de la realidad no admiten la existencia de axiomas no comprendidos en el sistema mismo (su software). En cambio, la mente humana puede percibir la verdad de los enunciados de cualquier sistema porque no está sujeta a esa determinación que existe en las máquinas y porque posee la flexibilidad que le otorga el maravilloso libre albedrío producto del objeto más complejo que conocemos de la creación: el cerebro humano. 
En tercer lugar, sigamos ahora el razonamiento de Gilbert Ryle en su texto de la edición 2003. Este autor planteó que Descartes incurrió en una equivocación fundamental referida a categorías lógicas, cuando estableció el dualismo. Tiene razón si no se toma en cuenta los fenómenos de la transducción que mediarían para hacer posible la distinción conceptual. No son equiparables materia y subjetividad sin la idea de emergencia que se transduce en la mente. Pero, el problema no está en categorías lógicas, porque va más allá del análisis filosófico y porque es un problema netamente científico que requiere el aporte de más especialistas, como físicos, bioquímicos, biólogos y neurocientistas.

Francis Crick, (1916-2004) quien es el científico que junto con James Watson descubrió el ADN y cambió para siempre la biología, ha reflexionado sobre el problema y señala que la visión es un buen punto de partida para el análisis de la búsqueda científica de la mente. La idea de Crick (2003) es que la visión es una demostración de la emergencia que lleva a la creación de la mente. Pasamos de la materia, en este caso biológica, a la mente, alma o espíritu o como queramos llamarla. Al parecer, Descartes tenía razón, no cometió ningún error categorial: el cerebro produce a la mente, son dos entidades distintas: materia y subjetividad. La sustancia extensa (la materia) produce efectivamente a la sustancia inextensa (la mente). Si alguien se equivocó, es justamente Ryle y no Descartes. Por lo demás, resulta muy extraño que un lógico de la calidad de Descartes se confundiera con un principio tan evidente como el de la categorización de los problemas científicos.

Por otro lado, si somos incapaces de resolver por ejemplo cuestiones como la planteada por Turing, entonces, ¿cómo desarrollar algoritmos tan complejos e infinitos como los necesarios para crear una mente artificial que dé a una máquina robot la plena autonomía en cuanto a decisiones y libre albedrío?

¿Podría, estimado doctor dar su opinión sobre las objeciones que he dado y en especial la referida al descubrimiento de Turing?

FJ: Bueno, si hemos de hablar de un reduccionismo necesario y conveniente para comprender el problema del libre albedrío y la plena autoconsciencia de soporte -desarrollada esta última a través de procesos biológicos-, el gran maestro fue Alan Turing, cuya obra se focaliza en el modelado matemático al interior de la muy especializada biología matemática, como el creador de la teoría de la Morfogénesis. El reduccionismo como clave para la comprensión: "Nuestro cerebro necesita que las cosas sean lo más simples posibles, pero no más simples, como diría Albert Einstein" (VILLATORO, 2010, párr.3).

Mediante ecuaciones de reacción-difusión Turing modeló los procesos de cómo se generan patrones durante el desarrollo de un embrión. A pesar del salto cualitativo que esto significó desde el punto de vista epistemológico, los biólogos experimentales todavía lo ven como un trabajo que no 
les significa:

Para estos últimos la realidad del laboratorio es tan compleja que un modelo tan sencillo no la puede explicar. Según Kondo y Miura $^{8}$ están equivocados. Solo se trata de un malentendido en el que los investigadores experimentales tienden a sucumbir. Los fenómenos biológicos más complejos tienen mecanismos subyacentes que son, en esencia, sencillos. Todo lo complejo emerge de lo sencillo. Las ecuaciones de reacción-difusión son una herramienta clave para el análisis teórico de toda la complejidad observada en la generación de patrones gracias a las tecnologías ómicas. [...] La morfogénesis no es solo responsable de la formación de patrones en la pigmentación de los seres vivos. También son responsables de la asimetría izquierda-derecha en los vertebrados, el desarrollo de las extremidades, la ramificación de los pulmones y del sistema circulatorio, etc. (VILLATORO, 2010, párr.4, párr.6)

Según esto, doctor, las posibilidades del desarrollo de la inteligencia artificial implantada en cuerpos robóticos son enormes...y son fascinantes...

CV: Habiendo oído su opinión sobre los argumentos anteriores creo que puede existir un desánimo sobre el futuro y mejor aún, sobre los límites de la inteligencia artificial. Sigo manteniendo mis opiniones sobre esos límites.

FJ: ¿Sabe? ... de lo que usted dice, me surge una pregunta, porque lo que ha sostenido hasta ahora es que las máquinas necesitarían desarrollar una mente para adquirir consciencia y por lo que usted parece, está convencido que hasta ahora el desarrollo de la I-A es bastante pobre en sus logros. Mi pregunta se refiere a que si necesariamente las máquinas requieren de una mente como la nuestra. La misma evolución nos da ejemplos de existencia de animales que sin una mente son capaces de realizar tareas muy complejas, como ocurre en las abejas, hormigas y termitas. Quizás lo mismo pueda suceder con las máquinas, la consciencia podría emerger sin necesidad de una mente contenedora...

CV: Al parecer usted está generando con lo que me ha dicho nuevas preguntas y ellas se refieren a si las máquinas inteligentes podrían llegar a existir en una forma distinta a la biológica natural y, si podemos comparar a la materia viva, sometida a la evolución darwiniana, con las máquinas...

Creo que las máquinas con mente y consciencia podrían ser parte de la evolución darwiniana, pero no de las máquinas sino de nosotros, los humanos. Ahora, si las máquinas pudiesen empezar por ellas solas a fabricar generación tras generación de máquinas sin la intervención humana, ¿Podríamos hablar también de evolución darwiniana fuera de los seres vivientes?

FJ: De acuerdo, hay diferencias importantes entre los seres vivos y las máquinas y así lo reconozco. Pero antes de contestar a esa pregunta que es crucial para mi argumentación a favor de la posibilidad de contar, en el futuro aún lejano, con máquinas autónomas independientes del ser humano, veamos

${ }^{8}$ Kondo y Miura son los referentes más cercanos en la aplicación del trabajo de Turing

Revista Tópicos Educacionais, Pernambuco, v. 27, n. 02, p. 164-181, 2021. ISSN: 2448-0215.

https://periodicos.ufpe.br/revistas/topicoseducacionais/index

DOI: $10.51359 / 2448-0215.2021 .249287$ 
algunos hechos que ocurren en seres vivos robotizados (con programas instalados en su genética)... Volviendo a los insectos como las hormigas y termitas, construir una vivienda social no es solamente actuar en común para dedicarla a la reproducción y el cuidado de los nuevos miembros, sino que tiene que haber una inteligencia distinta a la que conocemos en nosotros, dirigida a la preservación de la especie y su permanencia en el tiempo. Por inteligencia desde la etología entendemos la capacidad de actuar de manera de preservar a la especie; no nos referimos a ningún aspecto cognitivo ni mucho menos. Esa inteligencia es capaz de mantener funcionando a la colonia de manera de alcanzar determinados logros biológicos y sociales... creo que para las máquinas deberá definirse otro itinerario evolutivo; la evolución darwiniana es la proposición de un hombre para los hombres.

CV: Estimado doctor, como puede deducirse de lo que ha expresado, cualquier ser vivo a partir de los insectos posee más complejidad y mayores funciones que los robots que ahora tenemos. Siempre que se considere a seres animados ${ }^{9}$ no sintientes como robots.

Pero de allí en adelante la metáfora de los robots con programación ciega no funciona porque emergen funciones como el aprendizaje que nos alejan de la hipótesis y hay que buscar nuevas hipótesis explicativas. Las funciones humanas, como la capacidad de tener consciencia del fluir del tiempo y mantener un registro del pasado y una prospección de él usando los datos disponibles del presente y pasado. $\mathrm{O}$ sea, la vida consciente es producto de la mente, que tiene la particularidad de ser una entidad abstracta que se produce en la materia.

Tenemos una ontología de la mente. La mente se desarrolla según un plan o esquema genético, en el sentido biológico, de potencialidades. En cuanto a teorías sobre la mente y lo que ésta es, Freud hizo un aporte coherente desde el psicoanálisis, Finalmente, las construcciones psicológicas organizadas podríamos llamarlas mente.

Al comienzo señalamos que ha sido un error muy común de parte de los investigadores el estudiar separadamente a los fenómenos mentales que estamos discutiendo, olvidando que la mente, la consciencia, el pensamiento y la inteligencia son holísticos en esencia, no existen unos sin los otros ni podemos estudiar el cerebro por partes si queremos entender el psiquismo en toda su amplitud y complejidad.

FJ: Permítame una breve interrupción. ¿Podría usted darnos una definición de lo que entiende por consciencia?

CV: Trataré de explicar lo que entiendo por el término. Consciencia es para mí, la capacidad de autoconocimiento diferenciador del medio y de los demás. Es una propiedad emergente de la mente;

${ }^{9}$ ¿Podríamos clasificar en una eventual taxonomía a los robots conscientes como seres vivos?

Revista Tópicos Educacionais, Pernambuco, v. 27, n. 02, p. 164-181, 2021. ISSN: 2448-0215.

https://periodicos.ufpe.br/revistas/topicoseducacionais/index

DOI: 10.51359/2448-0215.2021.249287 
es la máxima emergencia. Es la expresión de una super-mente, es la organización de todos los procesos mentales; es una creación subjetiva de la materia: es la transducción de la materia hacia un estado de subjetividad que surge de la evolución y es un paso anterior al de la inteligencia. Es el gran conector de todos los procesos psíquicos que se dan en la mente. Es un espejo de la realidad externa en donde nos miramos y reconocemos como entidad pensante, con discurso y cognoscente. Contiene a la sensibilidad, lo que nos hace íntegramente consciente de nuestros sentidos, la sensitividad al medio y la emocionalidad. La reflexibilidad de la mente es el proceso y estado que originó a la autoconsciencia.

Entiendo que esta definición es bastante larga, pero el fenómeno que tratamos de entender es a su vez tan enormemente complicado que no basta con una o dos oraciones para expresar toda esa complejidad. Por algo el proceso evolutivo necesario para el desarrollo de la consciencia ha sido tan enormemente largo.

En los animales no conscientes, la mente es el espejo que refleja la realidad; cuando existe consciencia, hay un paso evolutivo nuevo: la mente se refleja en ese espejo que retiene la realidad externa y nos miramos en él: nos vemos y nos reconocemos y tratamos de comunicarnos y de entender a otras mentes. Todo en un proceso holístico, en que confluyen y se mezclan mente, consciencia e inteligencia. Por eso pensamos en varios planos a la vez y avanzamos o retrocedemos en nuestro tiempo mental no una sino miles de veces en el día. Recuerdo unas frases del Eclesiastés (1:6) que me recuerdan al trabajo mental: "El viento va al mediodía, y rodea al norte; va rodeando de continuo, y por sus rodeos vuelve el viento de nuevo hasta completar su ciclo

Para completar el análisis, tenemos que hacer una separación conceptual: hay que distinguir entre consciencia y autoconsciencia. La primera se refiere al darse cuenta, al tomar nota de lo externo al ser. La segunda, perteneciente a los animales superiores, se refiere al tomar nota de la primera persona, que se ve evolutivamente y tal como ocurre en los primates, que fácilmente entienden la idea del Yo al aceptar su nombre y reconocer el que les damos a los otros. Según Nagel (1974), el argumento de la primera persona es distinto a la posición epistemológica que se da desde la tercera persona, que podría ser descriptiva de los estados de consciencia que ocurren en un robot, por ejemplo.

FJ: Parece que ahora tenemos una definición de consciencia, profesor. Lo que nos falta definir es conocimiento, porque hay una relación y una interdependencia entre los conceptos de mente, consciencia, aprendizaje, conocimiento e inteligencia, todas cualidades emergentes de la materia (¿o del espíritu generado por la materia?). Por otra parte, el conocimiento plantea nuevos problemas, toda vez que el material genético incluye conocimientos que pasan directamente a los descendientes y un caso extremo está en los insectos que traen toda su conducta rígidamente empaquetada en los 
genes. Ese saber, ¿cómo se transduce y se transforma en una conducta, es decir, en un movimiento coordinado y con un fin, que llamamos comportamiento? Esta idea nos lleva a otro paso en la evolución de los robots mecánicos, distintos de los robots biológicos como los insectos y las bacterias, que están determinados en su existencia al logro de la preservación de la especie, mediante la reproducción que permite la recombinación genética y por tanto a la evolución darwiniana.

También hay que tomar en consideración que es extraño, con todas las posibilidades evolutivas que se han dado en el planeta, que la consciencia reflexiva surgiera solamente una vez en cuatro mil quinientos millones desde el surgimiento de la vida; es un logro tan extraordinario, tan único y quizás tan irrepetible que no sea frecuente que se produzca muchas veces en el universo, pese a la enormidad de éste y su prodigiosa antigüedad de más de 14 mil millones de años y a los miles de millones de planetas semejantes ala Tierra que existen en él.

Respecto el origen de la vida, tal vez profesor sería bueno recordar algunas cosas que ya se han dicho:

\begin{abstract}
Maravillosamente disponemos del libre albedrío y no estamos sujetos a leyes rígidas en nuestro comportamiento, lo que nos permite disfrutar a voluntad del arte, de la poesía y del amor. [...] Como un día señalara el gran filósofo católico Jean Guitton, ¿El mismo Dios no es ya perceptible, reconocible, casi visible, en el fondo último de lo real que describe el físico? [...] Al conocer todas las limitaciones de la ciencia deberíamos tener una mente abierta ante la presencia de una inteligencia superior a la humanidad y que ha creado al universo con una legalidad que funciona perfectamente pero que no podemos entender completamente. Quizás los límites de la ciencia que conocemos sean solamente los límites de nuestra mente. (WALKER y CAMPOS 2017, pp. 46-47)
\end{abstract}

Quisiera ahora, su opinión acerca de lo que llamamos inteligencia en el psiquismo, no en las máquinas, y quisiera saber si es éste un concepto de los que usted llama indecidibles...

CV: Creo que para introducirnos en este tema debemos considerar que tenemos que tomar el problema desde la perspectiva holística, en la metáfora darwiniana y puedo decir, doctor, que efectivamente la inteligencia es un término indecidible porque no hay una sola teoría aceptada por toda la comunidad, sino hay muchas teorías existiendo al mismo tiempo.

Como un producto evolutivo y en un contexto biológico, la inteligencia emerge o es un producto de la mente, lo que hemos observado antes. La inteligencia considerada evolutivamente, es el conjunto de factores de base cerebral que permite la sobrevivencia de la especie y, por lo tanto, del individuo. Esto es más difícil de observar en un robot y aún en un cyborg, el nivel más avanzado. La inteligencia permite la comunicación, la conservación de recuerdos, memorias y procedimientos que mejoran cada vez más el dominio ecológico del planeta e impulsa a los genes 
para diseminarse en el espacio mediante el poblamiento de otros planetas...

FJ: Gracias profesor, creo que hemos desarrollado lo que vinimos a debatir. No será, por supuesto, la última vez...

\section{Conclusiones}

No hemos sido diseñados para ver la realidad a través de las máquinas y de su lógica absoluta. El problema cartesiano implícito podría resumirse en que la res extensa-el cuerpo- es un ente biológico y la res cogitans - la mente- es una entidad que es un producto de aquella. No hay dos entes separados. Es claro que es distinto con la I-A, donde no existe tal división categorial y donde no podemos esperar en tiempos similares las mismas funciones ni los mismos logros de la mente humana.

Muchas esperanzas se esperaron de la I-A, pero solamente ahora, luego de más de medio siglo de investigación, apenas contamos con robots programados, sumidos en una determinación que no permite lograr en sí mismos la flexibilidad de la mente e inteligencia humana. Una de las primeras propuestas de la I-A. fue la cooperación docente a través de programas computacionales de alta eficacia. Hasta ahora no hay ninguna máquina que aprenda por sí sola. En contraste, una abeja que, con apenas mil neuronas, es capaz de discriminar locaciones y desarrollar toda su vida productiva mediante una programación genética maravillosa.

Ambos sistemas -el biológico y el físico- no son reductibles entre sí porque su origen y complejidades son distintas y por lo mismo sus causas, efectos, causalidades y emergencias son diferentes: son irreductiblemente complejas. Que las máquinas pensarán, es una afirmación que contiene una predicción, pero, quizás no estamos haciendo las preguntas correctas. Las máquinas primero tendrán que tener una mente que contenga activamente recuerdos, experiencias, errores, estrategias para resolver problemas y autoconsciencia. La pregunta entonces es si podemos crear una mente en los robots. De otra manera seguirán siendo pobres imitaciones de nosotros.

Es cuestión de técnica y de tiempo que la evolución se ocupe de las máquinas; ese momento comenzará cuando ellas sean capaces de autocopiarse y auto- mejorarse para que cada nueva generación aumente sus capacidades cognitivas e intelectuales. No hay nada en la naturaleza que impida el surgimiento de la auto-reflexibilidad si surge el pensamiento. Ahora, si consideramos la conservación de la especie, biológica o robótica, como parte de la inteligencia, entonces también deberíamos considerar la autoconservación agresiva en desmedro de otras especies que puedan rivalizar en el nicho ecológico, como parte de ella. Un pensamiento lógico- matemático absoluto no tiene ninguna de las expresiones emocionales con que nos dotó la naturaleza. Los valores se asocian Revista Tópicos Educacionais, Pernambuco, v. 27, n. 02, p. 164-181, 2021. ISSN: 2448-0215.

https://periodicos.ufpe.br/revistas/topicoseducacionais/index 
siempre a componentes emocionales y como decía Kant, deben surgir de la propia racionalidad, pero ¿cómo pueden surgir valores ajenos a la emocionalidad?

Quizás, en otras partes del universo ya hay evolución en las máquinas, pero en estos momentos, en nuestro planeta, con pocas décadas de experimentación e investigación, aún está muy lejana esa posibilidad. Tendremos cada vez más autómatas eficientes y complejos, pero si aún no sabemos mucho acerca de nuestra propia mente, de nuestra consciencia y de nuestro pensamiento, ¿cómo replicar esas propiedades psíquicas en las máquinas?

\section{Referencias}

CAMPOS, Nelson. Psicobiología de la percepción visual. Santiago de Chile: FCB, 2006.

CRICK, Francis. La búsqueda científica del alma. Madrid: Debate, 2003.

DAMASIO, Antonio. El error de Descartes. Madrid: Crítica, 2001.

GARRIDO, Juan. Emergencias del ser: la finitud del ser en Fichte y Heidegger. Revista de Filosofia Eikasia Universidad de Sevilla, dic., pp. 313-324, 2015.

GEYMONAT, Ludovico. Historia de la filosofía y de la ciencia. Madrid: Crítica, 2009.

GONZÁLEZ, Rodrigo. El test de Turing: dos mitos, un dogma. Revista de Filosofía, 63, pp. 37-53. http://dx.doi.org/10.4067/S0718- 43602007000100003, 2007.

KONDO, Shigeru; MIURA, Takashi. Reaction-Diffusion Model as a Framework for Understanding Biological Pattern Formation. Review Science, 329, pp.1616-1620. DOI: 101126/science.1179047, 2010.

NAGEL, Thomas. What is it like to be a bat? Philosophical Review, Duke University press, 83, pp. 435-450. DOI: 10.2307/2183914, 1974.

PÉREZ, Aristóbulo et al. Observaciones conductuales en el viaje de Darwin a bordo del Beagle. Revista Latinoamericana de Psicología, 39(3), pp. 503-521, 2007.

RYLE, Gilbert. The concept of mind. Chicago: University of Chicago Press. DOI: 10.7208/chicago/9780226922652.001.0001, 2003.

WALKER, Walter; CAMPOS, Nelson. Los teoremas de Gödel y la epistemología actual de las ciencias sociales. Santiago de Chile: Ediciones UCSH, Cuadernos de Ideas, 2017.

\section{Referencias de Soporte Informático}

ALONSO, José Ramón. Descartes y el dualismo cuerpo-mente. Blog. 18 mar. Disponible en: https://jralonso.es/2014/03/18/descartes-y-el-dualismo-cuerpo-mentel

MADRUGA, Alejandro. Inteligencia artificial fuerte. Blog 05 mar. Disponible en: https://cibersociedad.wordpress.com/2007/03/05/inteligencia-artificial-fuerte/ 
MORATA, Ginés. El siglo del gen. Biología molecular y genética. OpenMind Ab. Disponible en: https://www.bbvaopenmind.com/articulo/el-siglo-del-gen-biologia-molecular-y-genetica/, 2014.

VILLATORO, Francisco. Alan Turing, el genio matemático que creó la teoría de la morfogénesis poco antes de suicidarse. Blog 24 sept. Disponible en: francis.naukas.com/.../alan-turing-el-geniomatematico-que-creo-la-teoria-de-la-morf...., 2010.

Recebido em 12 de janeiro de 2021. Aprovado em 09 de outubro de 2021. 